\title{
Review
}

\section{Polyamorphism in water}

\author{
By Osamu Mishima*1,†
}

(Communicated by Hiroo INOKUCHI, M.J.A.)

\begin{abstract}
Water, the most common and important liquid, has peculiar properties like the density maximum at $4{ }^{\circ} \mathrm{C}$. Such properties are thought to stem from complex changes in the bonding-network structure of water molecules. And yet we cannot understand water. The discovery of the high-density amorphous ice (HDA) in 1984 and the discovery of the apparently discontinuous change in volume of amorphous ice in 1985 indicated experimentally clearly the existence of two kinds of disordered structure (polyamorphism) in a one-component condensed-matter system. This fact has changed our viewpoint concerning water and provided a basis for a new explanation; when cooled under pressure, water would separate into two liquids. The peculiar properties of water would be explained by the existence of the separation point: the liquid-liquid critical point (LLCP). Presently, accumulating evidences support this hypothesis. Here, I describe the process of my experimental studies from the discovery of HDA to the search for LLCP together with my thoughts which induced these experiments.
\end{abstract}

Keywords: Polyamorphism, water, amorphous ice, liquid-liquid transition, liquid-liquid critical point, liquid and glass

\section{Introduction}

In the 17th century, Galileo thought ice should be rarefied water. This stimulated the experimentalists of "Academie del Cimento". They cooled water, measured its volume and observed the density maximum. ${ }^{1}$ In spite of the long history of the succeeding water researches, there has been no clear explanation for this peculiar behavior (Fig. 1). In comparison with gas and crystal, we should admit our poor understanding of liquid, especially water.

Water freezes to crystalline ice $\mathrm{I}_{h}$ below $0{ }^{\circ} \mathrm{C}$, but water can also be supercooled, keeping the liquid state. The basic reason why water is difficult to understand is because the supercooled water crystal-

*1 National Institute for Materials Science, Tsukuba, Ibaraki, Japan.

$\dagger$ Correspondence should be addressed: O. Mishima, Polyamorphism Group, Exploratory Nanomaterials Research Laboratory, National Institute for Materials Science, 1-1 Namiki, Tsukuba, Ibaraki 305-0044, Japan (e-mail: MISHIMA.osamu@nims.go.jp).

Abbreviations: LDA: low-density amorphous solid; HDA: highdensity amorphous solid; LDL: low-density liquid; HDL: highdensity liquid; LLT: liquid-liquid transition; LLCP: liquid-liquid critical point; NML: no-man's land (the pressure-temperature domain of easy crystallization). lizes easily between $\sim 150 \mathrm{~K}$ and $\sim 235 \mathrm{~K}$ (Fig. 2). The temperature domain of the easy crystallization was named "no-man's land" (NML). Consequently, measurement of the liquid water in this NML is technically difficult. When temperature is decreased from $4{ }^{\circ} \mathrm{C}$, the volume of water increases from $1 \mathrm{~cm}^{3} / \mathrm{g}$ (or the density decreases from $1 \mathrm{~g} / \mathrm{cm}^{3}$ ) and it shows the tendency to become infinite (or zero) at $\sim 228 \mathrm{~K}$ in NML. ${ }^{2)}$ However, what really occurs in the liquid water of NML is unknowable.

At temperatures lower than $\sim 150 \mathrm{~K}$, the existence of the low-density amorphous ice (LDA) has been known since 1935. LDA was made by the deposition of water vapor onto a cold substrate ${ }^{3)}$ or by the extremely rapid cooling, $\sim 10^{6} \mathrm{~K} / \mathrm{s}$, of micrometersize droplets of liquid water.4) Water molecules of LDA were located disorderly like those of liquid water. However, LDA was solid, and its density, $\sim 0.94 \mathrm{~g} / \mathrm{cm}^{3}$, was as low as that of ice $\mathrm{I}_{h}$. Although we might regard LDA as vitrified water, ${ }^{5), 6)}$ the relationship between LDA and the high-temperature liquid water was unknown due to NML.

Incidentally, there was a natural expectation that the disordered structure of liquid, or glass, would change gradually when pressure and tempera- 


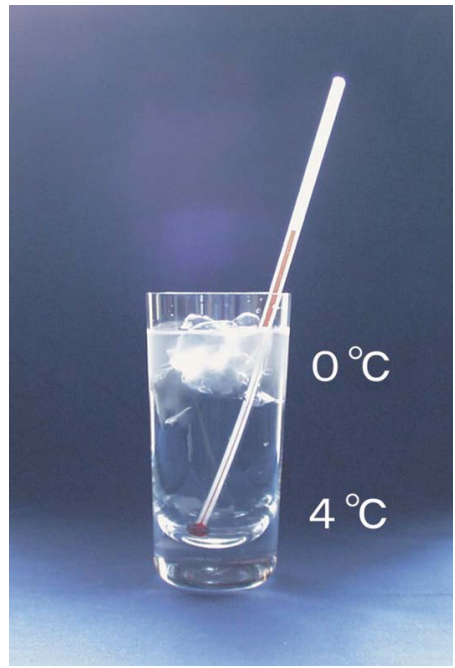

Fig. 1. Ice water in a glass. Cold liquid water floats on warmer water, which represents water's peculiar behavior.

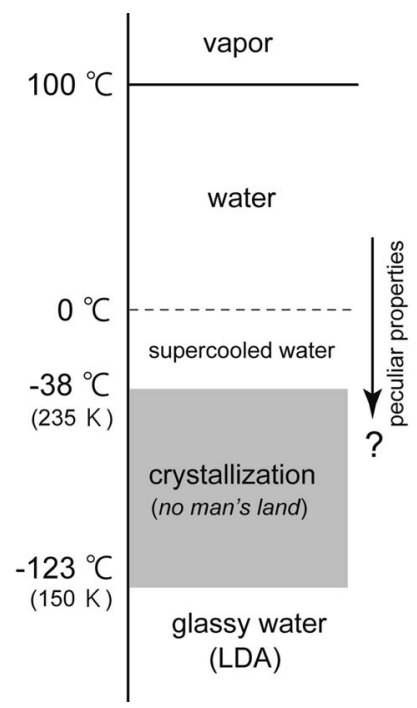

Fig. 2. The amorphous forms of $\mathrm{H}_{2} \mathrm{O}$ at one atmospheric pressure.

ture were changed. In other words, it was commonly thought that the one-component material would have only one liquid phase. The possibility of a discontinuous change in volume of liquid was doubted and rarely discussed because there was no clear experimental evidence for the amorphous polymorphism (or polyamorphism).

As described below, the discovery of the highdensity amorphous ice (HDA) at low-temperature and high-pressure conditions was the beginning of a novel and promising idea about the liquid state in NML.

\section{Pressure-induced amorphization: the discovery of HDA}

After Tammann reported in 1900 that the melting temperature of ice $\mathrm{I}_{h}$ decreased under pressure, the effect of pressure on melting interested the researchers of high-pressure phenomena of materials. ${ }^{7), 8)}$ As shown in Fig. 3a, Naoto Kawai of Osaka University thought that the pressure $(P)$-temperature $(T)$ line where a crystal would melt should continue to $\sim 0 \mathrm{~K}$ if the crystal was not transformed to other crystalline structures.9),10) I was a Kawai's student and was interested in his idea. Later, in the compression experiment of ice $\mathrm{I}_{h}$, we observed that the transition pressure to other high-pressure ices became higher at lower temperatures as shown by the line A in Fig. 3b. ${ }^{11)}$ Then, I noticed that ice $\mathrm{I}_{h}$ might be compressed to its expected melting line (B in Fig. 3b) at adequately low temperatures. Usually, melting occurred easily on the melting line. The definition of melting was vague, and it had once been regarded as instability of a crystalline structure. ${ }^{12)}$ Consequently, it was considered that, even if temperature was low, ice $\mathrm{I}_{h}$ would do some kind of transition when it was compressed to its melting pressure. This speculation became gradually my motivation to compress ice $\mathrm{I}_{h}$ at the liquid-nitrogen temperature.

At National Research Council of Canada in Ottawa where I stayed for a few years as a postdoctoral fellow, my speculation about the lowtemperature melting of ice $\mathrm{I}_{h}$ interested Whalley. We discussed ice $\mathrm{I}_{h}$ might become ('melt' into) a glass if temperature was lower than the glass-transition temperature $\left(T_{\mathrm{g}}\right)$ of liquid water. In order to examine this, I compressed ice $\mathrm{I}_{h}$ at $77 \mathrm{~K}$ by using a piston-cylinder apparatus. The volume $(V)$ of ice $\mathrm{I}_{h}$ decreased rapidly by more than $20 \%$ at $\sim 1 \mathrm{GPa}$ (Fig. 4). ${ }^{13)}$ When decompressed, the produced highdensity sample was recovered at one atmospheric pressure and $77 \mathrm{~K}$, and its density was $\sim 1.17 \mathrm{~g} / \mathrm{cm}^{3}$. The low-temperature X-ray measurement of the recovered solid sample by Calvert showed the halo diffraction ring, and this indicated the sample was apparently amorphous. The halo pattern resembled that of liquid water, which was consistent with the expectation of the pressure-induced 'melting'. Relatively easy production of this high-density amorphous ice (HDA), together with its reproducibility, 

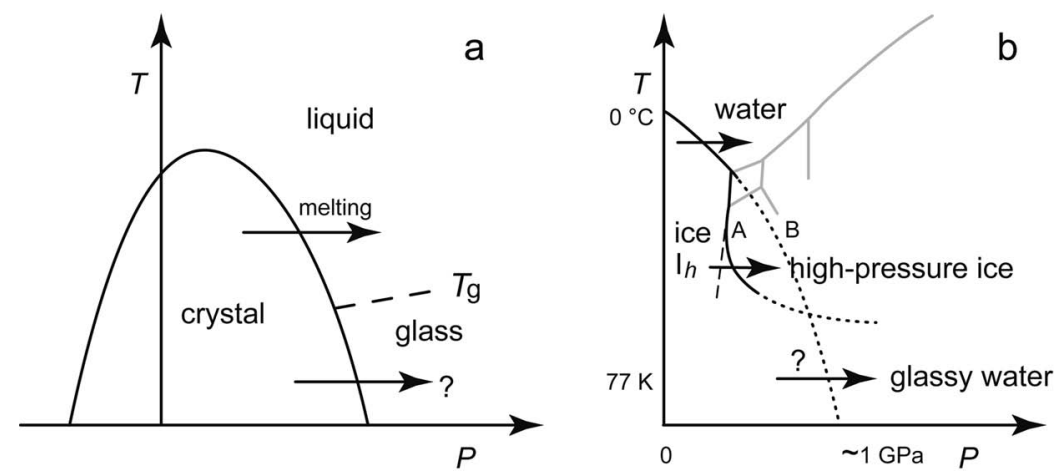

Fig. 3. The low-temperature melting of a crystal. a: a hypothetical general melting line. b: the phase diagram of $\mathrm{H}_{2} \mathrm{O}$. A: the line of the compression-induced crystal-crystal transition. B: the extrapolated melting line. One atmospheric pressure is $\sim 0.0001 \mathrm{GPa}$, or $\sim 0.1 \mathrm{MPa}$.

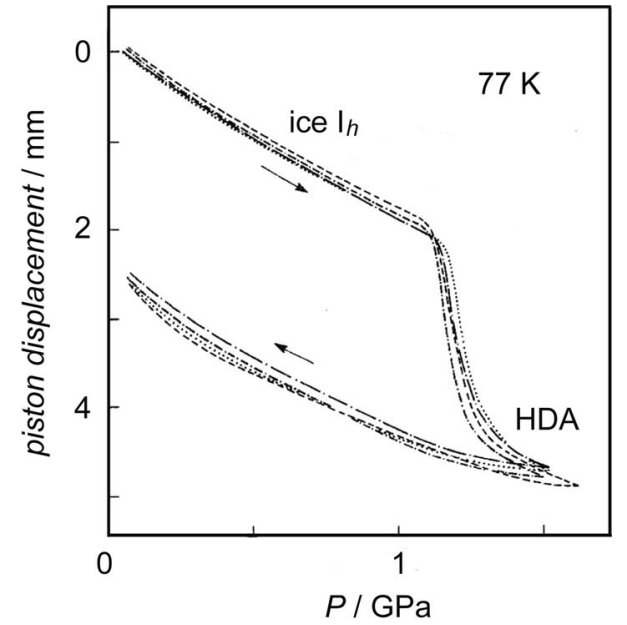

Fig. 4. The change in volume of ice $\mathrm{I}_{h}$ during the compression at $77 \mathrm{~K}^{13)}$

ensured its existence. The discovery of HDA was the discovery of a new method of making glass, and this initiated the research field of the pressure-induced amorphization of crystals. As shown in Fig 5a, I imagined the hypothetical potential surface from the Takeo Matsubara's idea about the pressure-induced melting which was illustrated in the Kawai's paper. ${ }^{10)}$ I speculated that the system ran out of the narrow hollow of ice $\mathrm{I}_{h}$ and that it immediately stopped due to both the roughness of the potential surface of the amorphous structure and the low temperature. I thought HDA was unstable and would relax toward the structure of the high-pressure liquid water.

\section{Transition between LDA and HDA: the discovery of apparent polyamorphism}

When HDA was heated at one atmospheric pressure, its structure relaxed gradually and HDA expanded slowly, and then, strangely, HDA quickly swelled by $\sim 20 \%$ at $\sim 120 \mathrm{~K}$ and changed into LDA (Fig. 6). When heated further, LDA crystallized into ice $\mathrm{I}_{c}$ at $\sim 150 \mathrm{~K}$, and then ice $\mathrm{I}_{c}$ changed to ice $\mathrm{I}_{h}$. When HDA was heated from $77 \mathrm{~K}$ at different pressures, it expanded gradually at low pressures and the expansion changed gradually toward contraction at high pressures (Fig. 6). On further heating under pressure, HDA crystallized into various high-pressure ices. Strictly speaking, the halo X-ray diffraction pattern of HDA indicated HDA was either truly amorphous or microcrystalline. However, I thought HDA was truly amorphous from this relaxation-crystallization manner.

In this experiment, the temperature of the rapid HDA-to-LDA transition showed strong pressure dependence; the temperature decreased when the pressure decreased. From this noteworthy fact, I thought that HDA would change rapidly to LDA if it were decompressed to negative pressures at $77 \mathrm{~K}$ (Fig. 6). Moreover, I supposed by analogy that the LDA, made by the low-temperature decompression, would be truly amorphous because HDA, made by the $77 \mathrm{~K}$ compression, was likely truly amorphous. Further, I expected conversely that when LDA was compressed, it might change to HDA rapidly at a certain pressure. This rapid LDA-to-HDA transition was expected also because the structure of LDA seemed 
a

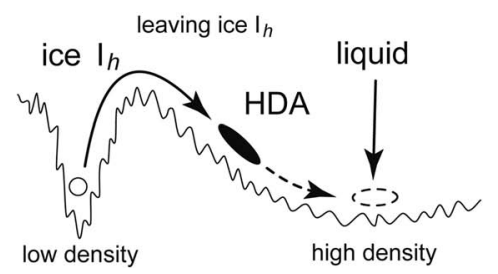

b

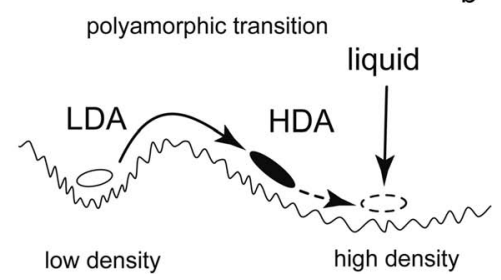

Fig. 5. The $\mathrm{H}_{2} \mathrm{O}$ states on the supposed potential surface. a: the amorphization, or melting. b: the polyamorphic transition.

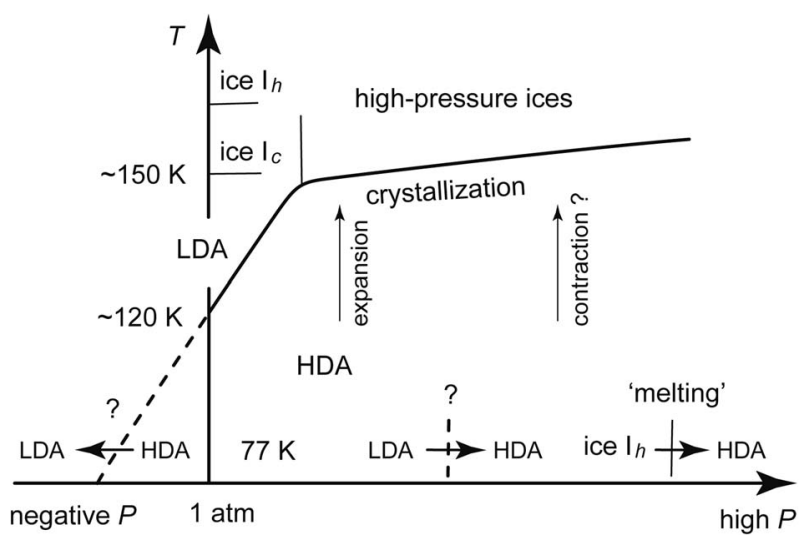

Fig. 6. The stability of HDA in the $P$ - $T$ diagram.

somewhat similar to the stable structure of ice $\mathrm{I}_{h}$ which showed the rapid change to HDA (Fig. 4). As shown in Fig. 5b, I imagined a basin of LDA and speculated that the system might run away out of the basin by compression. If so, there would be two amorphous phases of different disordered structures, and the transition between the two phases might occur discontinuously; the transition would be firstorder. The desire to examine the possibility of the two amorphous phases became my motivation to compress LDA at a low temperature.

In July 1984, I compressed LDA at $77 \mathrm{~K}$, and found that its volume decreased by $\sim 20 \%$ suddenly, rapidly and discontinuously at $\sim 0.6 \mathrm{GPa}$ (Fig. 7). The sharpness of the transition contrasted remarkably with the known dullness of the pressure-induced densification of general glasses. ${ }^{14)}$ The transition pressure of $\sim 0.6 \mathrm{GPa}$ was much lower than that of $\sim 1 \mathrm{GPa}$ of ice $\mathrm{I}_{h}$, which hinted that LDA was truly amorphous. Then, the halo pattern of LDA before the transition and the halo pattern of HDA after the transition were confirmed by the x-ray measurements of the recovered samples (Fig. 8). This LDA-HDA transition was the first experimental example that clearly indicated the existence of polyamorphism. Whalley recognized the importance of this transition immediately, and we published it. ${ }^{15)}$

\section{Water's liquid-liquid transition and its liquid-liquid critical point}

The discontinuous LDA-HDA transition implied the validity of the two-basin picture of Fig. 5b. Accordingly, if LDA and HDA were heated to temperatures higher than their $T_{\mathrm{g}} \mathrm{s}$, they were thought to relax into low-density liquid (LDL) and highdensity liquid (HDL), respectively. Moreover, the discontinuous liquid-liquid transition (LLT) between LDL and HDL and the point where this LLT would disappear, the liquid-liquid critical point (LLCP), were imagined naturally. Liquid would change continuously at temperatures higher than the LLCP. On the other hand, at temperatures lower than the LLCP, hysteresis of the first-order transition between compression and decompression cycles was thought to occur; the hysteresis would be larger at lower temperatures. These were speculations, but in 1992, Poole et al. proposed this LLCP hypothesis concretely by the molecular-dynamics simulation of supercooled water and by taking the discontinuous LDA-HDA transition into account. ${ }^{16)}$ Both our experimental fact of polyamorphism and the theoretical prediction by Poole et al. have directed my interest toward the experimental identification of the LLCP.

4.1. Experimental problem. The difficulty of the experimental proof of the LLCP hypothesis, apart from the crystallization in NML, was that the amorphous ices were solid and not in the thermodynamical equilibrium. As for liquid, it was in the equilibrium and could be a sole state once pressure and temperature were fixed. Therefore, it would be possible to prove the discontinuity of the transition between two different liquids. However, regarding 


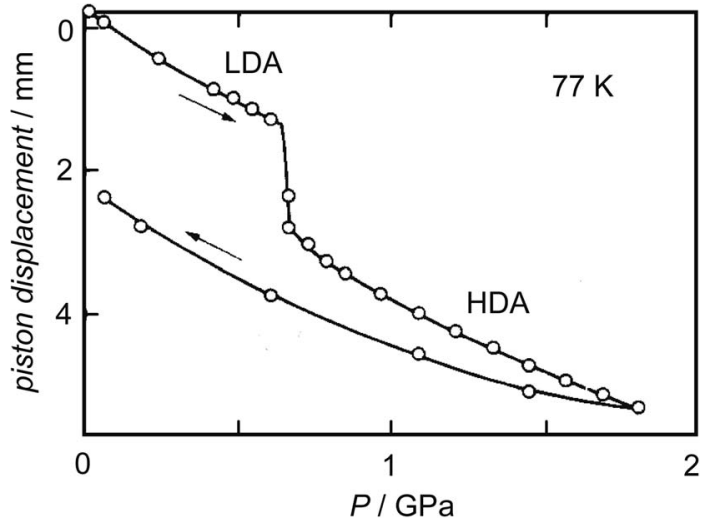

Fig. 7. The change in volume of LDA during the compression at $77 \mathrm{~K}^{15)}$

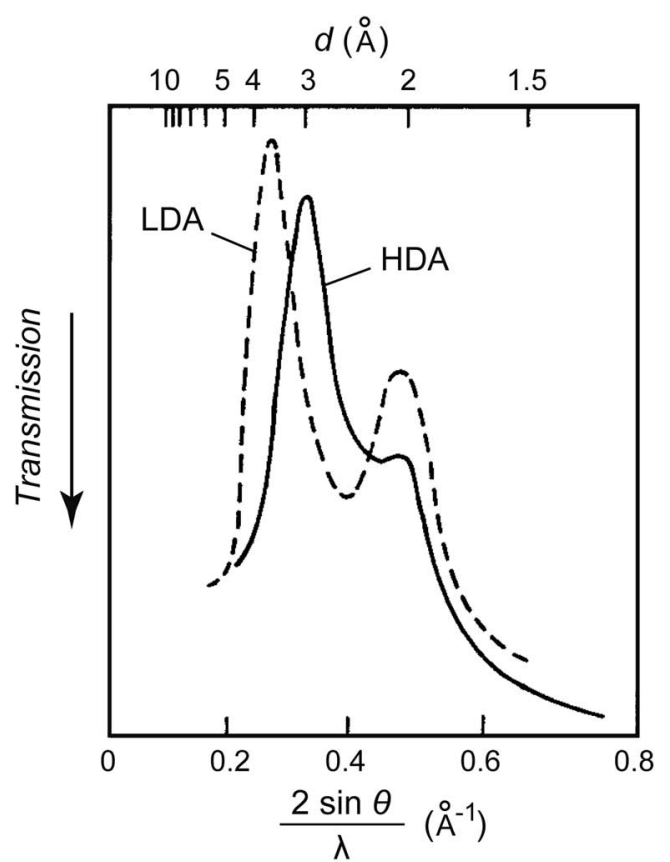

Fig. 8. X-ray diffraction patterns (micrometer tracing) of LDA (the broken line) and HDA (the solid line) at one atmospheric pressure and $\sim 95 \mathrm{~K}^{15}{ }^{15} 2 \theta$ : the scattering angle. $\lambda$ : the wavelength of the X-ray.

liquid water, LDL and HDL would crystallize immediately in NML, and we could not observe the LLT directly. In contrast, although we could observe the LDA-HDA transition, the non-equilibrium nature of LDA and HDA threw doubt on the discontinuity of the transition logically. That is, the existence of the barrier in Fig. 5b was doubted; the potential surface between LDA and HDA might be flat by nature.
Then, the apparently discontinuous LDA-to-HDA transition (Fig. 7) might be, correctly, continuous or caused by unknown sticky relaxation of a nonequilibrium LDA state. If so, LLT of water would be continuous and LLCP would not exist.

Besides, because the analysis of HDA was insufficient, it was argued that HDA was microcrystalline or that HDA and liquid water possessed different structures and were not related each other.

I started studying the discontinuity of the LDAHDA transition and the relationship between HDA and liquid water at National Institute for Researches in Inorganic Materials (now National Institute for Materials Science) in Tsukuba.

4.1.1. Discontinuity of the LDA-HDA transition. Slightly before the proposal of Poole et al., we squeezed directly LDA powder at $77 \mathrm{~K}$ between two diamonds of the so-called "diamond-anvil cell" (Fig. 9) and then heated the equipment from $77 \mathrm{~K}$. We watched the sample under pressure during the heating and observed the transition line between the low-pressure LDA and the high-pressure HDA visually. The existence of the line meant the existence of a difference in their refractive index, or density. The line became gradually sharper and more distinct while the amorphous ices relaxed by the heating until their crystallization at $\sim 150 \mathrm{~K}$. This showed clearly the existence of two amorphous phases up to the crystallization temperature. From this observation, we mentioned the possibility of a large and rapid change in density of compressed liquid water near $T_{\mathrm{g}}{ }^{17)}$ In a different study using a piston-cylinder apparatus, I measured the volume of LDA and that of HDA at different pressures and temperatures, as shown in Fig. 10. I confirmed the LDA-HDA transition was reversible and it had hysteresis. These results were consistent with the discontinuity of the transition. ${ }^{18)}$ Later, we kept a lump of HDA at a fixed temperature at one atmospheric pressure and observed the temporal HDA-to-LDA transition. The HDA-LDA boundary region propagated in the sample, and the width of the boundary region appeared to narrow simultaneously. ${ }^{19)}$ This hinted that the transition occurred discontinuously by cooperative movement of molecules.

4.1.2. Continuity between HDA and liquid water. As illustrated by the bold broken line of Fig. 11, the melting line of ice $\mathrm{I}_{h}$ became the amorphization line at low temperatures. Accordingly, HDL made by melting and HDA made by amorphization 


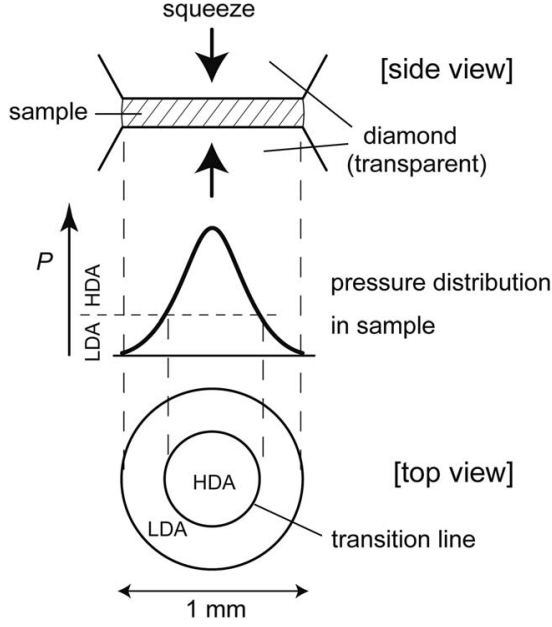

Fig. 9. Schematic illustration of visual observation of the LDA-HDA transition in the diamond-anvil cell.

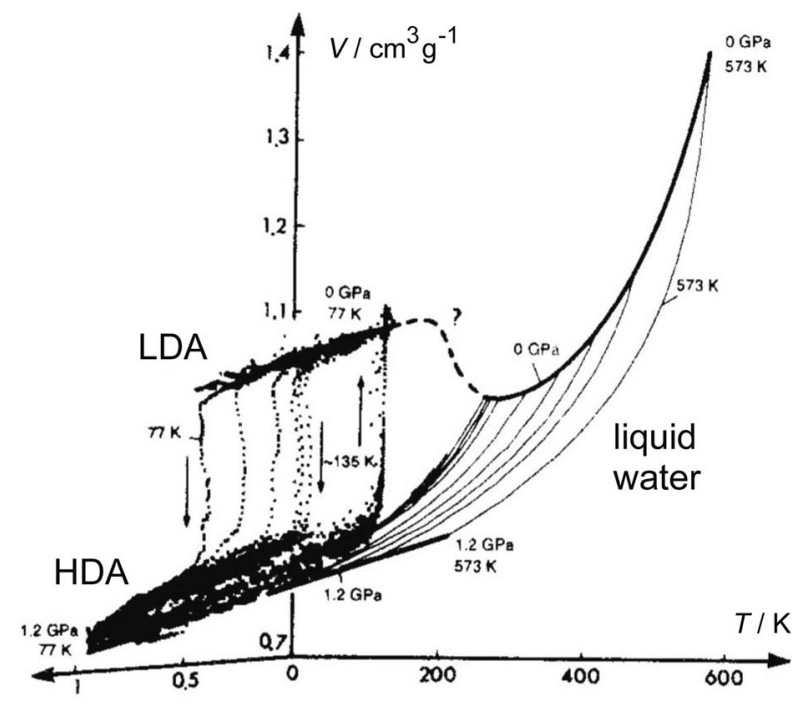

$\mathrm{P} / \mathrm{GPa}$

Fig. 10. The volume of amorphous forms of $\mathrm{H}_{2} \mathrm{O}$ at low temperatures. ${ }^{18)}$ The transitions between HDA and LDA are indicated by arrows.

were thought to connect directly around the supposed $T_{\mathrm{g}}{ }^{20)}$ Actually, this broken line was obtained by the compression of emulsified ice $\mathrm{I}_{h}$, that was the micrometer-size crystalline ice dispersed in oil. This experiment started as follows. It was known that the emulsified liquid water, or micrometer-size droplets of water, when cooled below water's freezing point, hardly changed into ice $\mathrm{I}_{h}$. From this, I wondered if it was difficult for the emulsified ice $\mathrm{I}_{h}$ to change to

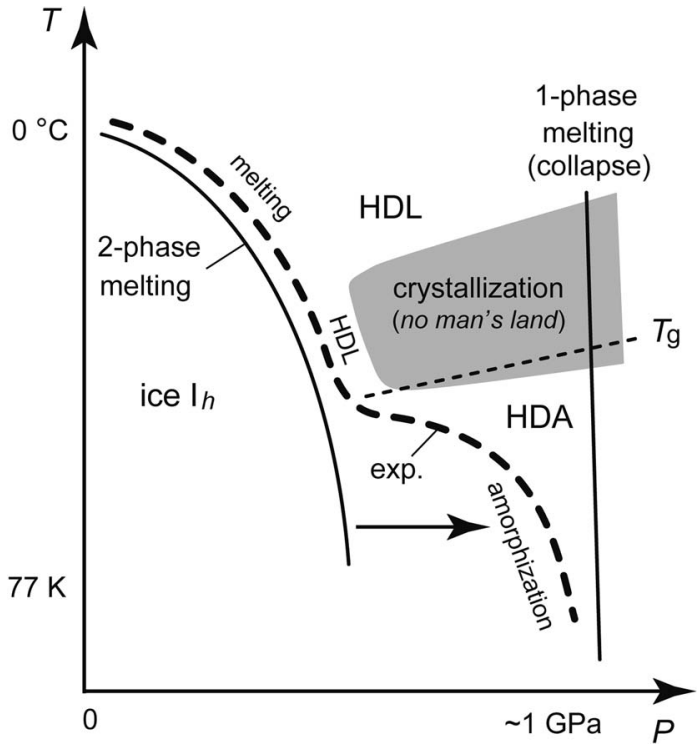

Fig. 11. Illustration of the relationship between melting and amorphization of ice $\mathrm{I}_{h}{ }^{20}$ ) The bold broken line, exp., is the experimental data.

other crystalline structures. In order to clarify this, I made the emulsified ice $\mathrm{I}_{h}$ by the crystallization of emulsified liquid water in NML and compressed it. It did not change to other crystalline phases on the line A of Fig. 3b, but it melted to the supercooled high-density liquid water (HDL) on the melting line $\mathrm{B}$ in the figure. Inside the NML, it was considered that the HDL, produced by the melting of ice $\mathrm{I}_{h}$, crystallized to high-pressure ices immediately (Fig. 11). On the other hand, when compressed at $77 \mathrm{~K}$, the emulsified ice $\mathrm{I}_{h}$ amorphized to $\mathrm{HDA}$ at the same pressure as the usual ice $\mathrm{I}_{h}$ amorphized. When the compression of the emulsified ice $\mathrm{I}_{h}$ was repeated at different temperatures, the melting line and the amorphization line were connected into one line. It was considered that the equilibrium "two-phase melting" between ice $\mathrm{I}_{h}$ and water changed continuously toward the "one-phase melting" (or collapse) of ice $\mathrm{I}_{h}$ at temperatures lower than $T_{\mathrm{g}}$ (the arrow in Fig. 11).

Later, we cooled the emulsified liquid water rapidly, $\sim 10^{3} \mathrm{~K} / \mathrm{s}$, under pressure, vitrified it and decompressed the glassy water to one atmospheric pressure at $77 \mathrm{~K}$. When heated, the glass changed to LDA, and this behavior was the same as that of HDA. This suggested HDA made by the pressureinduced amorphization resembled the vitrified HDL. ${ }^{21)}$ The existence of the glass transition from HDA to 

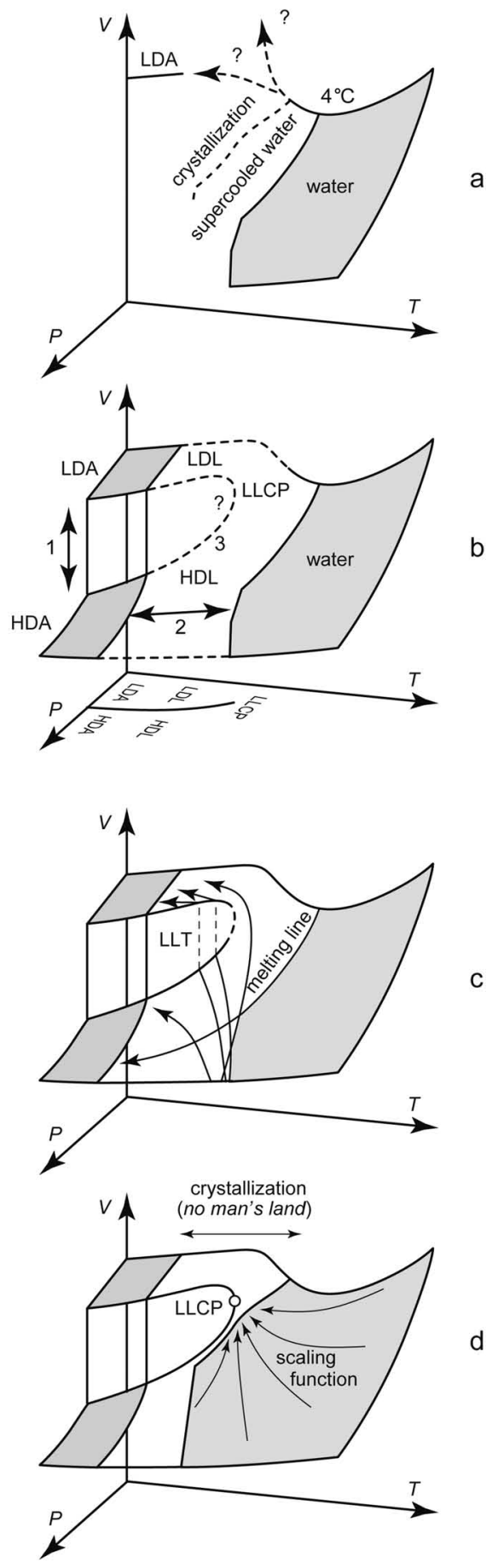

Fig. 12. The $P-V-T$ relationship between liquid water and amorphous ices obtained by experiments (the lines and the gray regions). a: before our studies. b: this figure corresponds to Fig. 10. c: the arrows are the melting lines of crystalline ices, suggesting the existence of liquid water along the lines (see Figs. 11 and 14). d: estimation of the location of LLCP by adjusting the scaling function.
HDL was also hinted from the analogy of the glass transitions of dilute $\mathrm{LiCl}$-aqueous solutions under pressure. 22 )

4.1.3. My view on the problem. From these evidences, I believed the discontinuity of the LDAHDA transition (the arrow-1 of Fig. 12b) and the thermodynamic connection between HDA and liquid water (the arrow-2 of Fig. 12b). This was the reason why I believed the LLCP hypothesis shown by the broken line-3 of Fig. 12b. The hypothesis seemed the simplest explanation for these experimental results. However, these evidences did not offer decisive proofs and, therefore, confusion of explanations existed. Although we could not affirm the discontinuity between LDA and HDA, and although some researchers suspected that HDA, the collapsed ice $\mathrm{I}_{h}$, might be microcrystalline, I began the experiments of the supercooled liquid water in order to search for the hypothetical LLCP (or paradoxically for a clear disproof of LLCP). Here, I always used the emulsified sample to hinder both the crystallization of the liquid and the crystal-crystal transition. The surfactant of the emulsion hardly dissolved in water, and the thermodynamic data of the emulsified water were practically the same as those of pure water in the region of overlap.

4.2. The location of LLT. Although we could not study the supercooled water in NML directly, the low-temperature metastable melting lines of the micrometer-size crystalline ices could be measured as demonstrated in Fig. 11. As shown in Fig. 13, if LLT existed, and if the LLT line intersected with such a melting line, the slope of the melting line had to change discontinuously because the manner of melting would be different between LDL and HDL. Therefore, the metastable melting line of the emulsified ice IV, a high-pressure ice, was measured. It was found that the slope of its melting line was discontinuous at a point on the theoretically expected LLT line. Consequently, the existence of LLT, or the existence of the two liquid phases, was suggested indirectly. ${ }^{23)}$ Meanwhile, Stanley invited me to write together a review article about the relationship between the liquid water and the two amorphous ices, and we co-authored it. ${ }^{24)}$

4.3. The location of LLCP. Contrarily, some researchers thought that the slope of the melting line of ice IV might curve strongly and continuously if the experiment were carried out much more precisely. Therefore, even if the change in the slope 


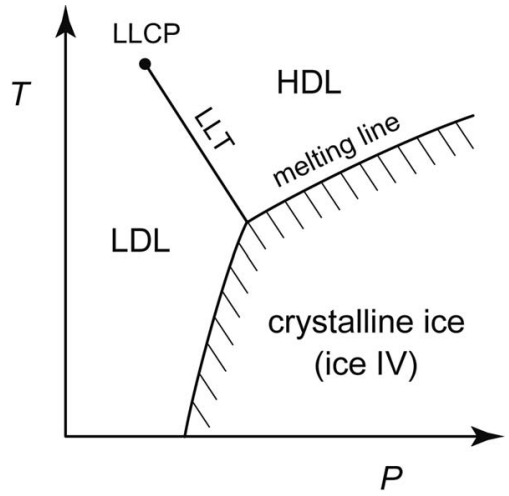

Fig. 13. Illustration of the discontinuous change in the slope of a melting line.
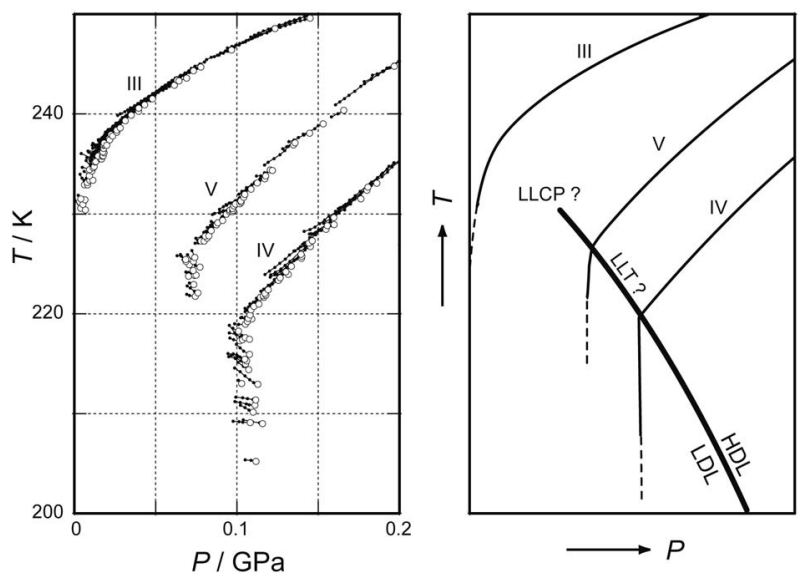

Fig. 14. The melting lines of emulsified $\mathrm{D}_{2} \mathrm{O}$ ices, III, IV and V. ${ }^{25}$ ) The almost vertical melting lines suggested small entropy of LDL.

appeared experimentally discontinuous, the discontinuity was doubted logically because experimental errors were always inevitable. Nevertheless, I measured the melting lines of other high-pressure ices. Then, I hinted the location of LLCP experimentally (Fig. 12c). In Fig. 14 are shown the melting lines of $\mathrm{D}_{2} \mathrm{O}$ ices. The melting lines of ice IV and ice $\mathrm{V}$ appeared to bend suddenly at $\sim 220 \mathrm{~K}$ and $\sim 225 \mathrm{~K}$, respectively. In contrast, the melting line of ice III continued smoothly to low pressures. This suggested that LLT disappeared around $\approx 50 \mathrm{MPa}$ and $\approx 230 \mathrm{~K}$; the LLCP of $\mathrm{D}_{2} \mathrm{O}$ might be located around here. ${ }^{25}$ ) Such melting lines were also used to estimate the water's Gibbs-energy surface that had LLT and LLCP (Fig. 15). ${ }^{23)}$

Even though it seemed almost impossible to de-

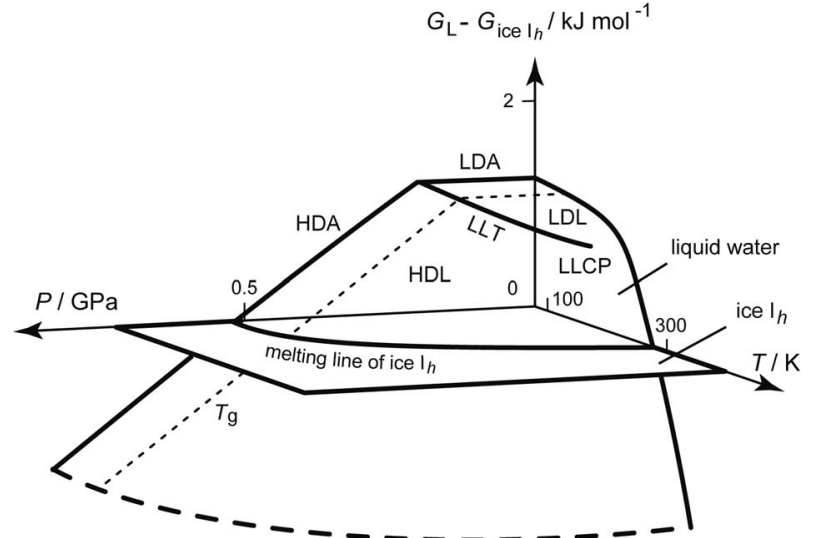

Fig. 15. The supposed difference between the Gibbs energy of liquid water, $G_{\mathrm{L}}(P, T)$, and that of ice $\mathrm{I}_{h}, G_{\text {ice } \mathrm{I} h}(P, T) .{ }^{23)}$ The hypothetical LLT and LLCP are drawn. The surface near LLCP might be described by the scaling function.

tect LLCP in NML directly by experiment, we might be able to suppose the location of LLCP by adjusting the scaling function, which described general critical phenomena, to supercooled water (Fig. 12d). A method for this was recently proposed, ${ }^{26}$ ) and I tried the method by using my new volumetric data of supercooled water. Then, the LLCP of $\mathrm{H}_{2} \mathrm{O}$ was located around $\approx 50 \mathrm{MPa}$ and $\approx 223 \mathrm{~K}$ (personal communications).

\section{Conclusion}

Our main results on water and amorphous ices are listed below and may be read in conjunction with Figs. 12b and 12c.

1) The discovery of HDA: the pressure-induced amorphization. ${ }^{13}$

2) The discovery of apparently discontinuous LDA-HDA transition: polyamorphism. ${ }^{15), 17)-19)}$

3) The experimental results consistent with the connection between HDA and liquid water. ${ }^{20)-22}$

4) The experimental results consistent with LLT. ${ }^{23}$

5) The experimental results consistent with LLCP. ${ }^{25)}$

Our discovery of the apparent polyamorphism in amorphous ice gradually and steadily inspired other researchers as if we have been awakened to the truth, and the polyamorphism formed a basis for the LLCP hypothesis. The hypothesis is strongly supported, but not proven, by the various evidences provided in these 25 years. The LLCP, if it exists, would affect the liquid state in a wide pressure- 
temperature region. As illustrated in Fig. 12, the minimum of volume (or the maximum of density) at $4{ }^{\circ} \mathrm{C}$ at one atmospheric pressure is explicable by the transformation of the high-temperature HDL toward the low-temperature LDL.

At least the following consensus seems emerging among water researchers. In the experimental aspect, the LDA-HDA transition is likely discontinuous, and the structure of HDA resembles that of our familiar liquid water (or HDL). ${ }^{27), 28)}$ In the theoretical aspect, almost all studies of the simulations support the LLCP hypothesis. ${ }^{29), 30)}$ The simulations can avoid the crystallization in NML and can provide information about the structure of liquid water. In addition, polyamorphism is found to arise generally from the 'wavy' pair potential between two model particles. $^{31), 32)}$ So far, the LLCP hypothesis has no serious defect although there are many unsolved questions such as the nucleation and growth of the LDA-HDA transition.

\section{Implications}

Polyamorphism is one of the basic concepts of the condensed-matter science. The polyamorphism in amorphous ice has stimulated the studies on the general polyamorphic transition, and these studies opened the broadened prospects on the researches of liquid and glass. ${ }^{33)-35)}$

Needless to say, water is related to various research fields. ${ }^{36}$ ) If we understand how the polyamorphism of water is affected by solutes and by the electromagnetic field etc., its knowledge may be useful to understand the phenomena like photosynthesis.

Polyamorphism was already applied to the experimental studies of aqueous solutions, water in narrow pores, ${ }^{37)}$ and water inside proteins. ${ }^{38)}$ Because these conditions sometimes hindered the crystallization of water in NML, such studies were used to examine the LLCP hypothesis and then to support it. An example was the liquid-liquid immiscibility of the dilute salt water at low temperatures, which was suggested about 40 years ago. ${ }^{39)}$ We presented evidences for the immiscibility by using LiCl-aqueous solutions and explained the immiscibility by the polyamorphism of pure water. ${ }^{40)-42)}$ As a result, $\mathrm{LiCl}$ was thought to dissolve in HDL than LDL. These studies would have implications for ice nucleation in clouds and in plants etc. ${ }^{43)}$ because the separated LDL readily crystallized.

Generally speaking, water represents liquid.
Therefore, the understanding of water may be the understanding of liquid. The notion of polyamorphism certainly changed the previous understanding of water qualitatively. About 350 years has passed since the experiment of "Academie del Cimento", and the existence of two kinds of liquid water, the secret behind the density-maximum phenomenon, is now gradually being revealed.

\section{References}

1) Waller, R. (trans.) (1684) Essayes of Natural Experiments (original in Italian by the Secretary of the Academie del Cimento): (1964) facsimile of the English translation. Johnson Reprint, New York.

2) Angell, C. A. (1982) Supercooled water. In Water: A Comprehensive Treatise. Vol. 7 (ed. Franks, F.). Plenum, New York, pp. 1-81.

3) Burton, E. F. and Oliver, W. F. (1935) The crystal structure of ice at low temperatures. Proc. R. Soc. Lond. A 153, 166-172.

4) Brüggeller, P. and Mayer, E. (1980) Complete vitrification in pure liquid water and dilute aqueous solutions. Nature 288, 569-571.

5) Sugisaki, M., Suga, H. and Seki, S. (1968) Calorimetric study of the glassy state. IV. Heat capacities of glassy water and cubic ice. Bull. Chem. Soc. Jpn. 41, 2591-2599.

6) Johari, G. P., Hallbrucker, A. and Mayer, E. (1987) The glass-liquid transition of hyperquenched water. Nature 330, 552-553.

7) Tammann, G. (1900) Ueber die grenzen des festen zustandes IV. Ann. Phys. 2, 1-31.

8) Bridgman, P. W. (1911) Water, in the liquid and five solid forms, under pressure. Proc. Am. Acad. Arts Sci. 47, 441-558.

9) Kawai, N. and Inokuti, Y. (1968) Low temperature melting of elements under high pressure and its progression in the periodic table. Jpn. J. Appl. Phys. 7, 989-1004.

10) Kawai, N. and Inokuti, Y. (1970) High pressure melting of general compounds, and with some physical models. Jpn. J. Appl. Phys. 9, 31-48.

11) Mishima, O. and Endo, S. (1980) Phase relations of ice under pressure. J. Chem. Phys. 73, 2454-2456.

12) Born, M. and Huang, K. (1954) Dynamical Theory of Crystal Lattices. Oxford Univ. Press, London.

13) Mishima, O., Calvert, L. D. and Whalley, E. (1984) 'Melting' ice I at $77 \mathrm{~K}$ and 10 kbar: a new method of making amorphous solids. Nature 310, 393-395.

14) Bridgman, P. W. and Šimon, I. (1953) Effects of very high pressures on glass. J. Appl. Phys. 24, 405-413.

15) Mishima, O., Calvert, L. D. and Whalley, E. (1985) An apparently first-order transition between two amorphous phases of ice induced by pressure. Nature 314, 76-78.

16) Poole, P. H., Sciortino, F., Essmann, U. and Stanley, H. E. (1992) Phase behavior of metastable water. Nature 360, 324-328.

17) Mishima, O., Takemura, K. and Aoki, K. (1991) 
Visual observations of the amorphous-amorphous transition in $\mathrm{H}_{2} \mathrm{O}$ under pressure. Science 254, 406-408.

18) Mishima, O. (1994) Reversible first-order transition between two $\mathrm{H}_{2} \mathrm{O}$ amorphs at $\sim 0.2 \mathrm{GPa}$ and $\sim 135$ K. J. Chem. Phys. 100, 5910-5912.

19) Mishima, O. and Suzuki, Y. (2002) Propagation of the polyamorphic transition of ice and the liquidliquid critical point. Nature 419, 599-603.

20) Mishima, O. (1996) Relationship between melting and amorphization of ice. Nature 384, 546-549.

21) Mishima, O. and Suzuki, Y. (2001) Vitrification of emulsified liquid water under pressure. J. Chem. Phys. 115, 4199-4202.

22) Mishima, O. (2004) The glass-to-liquid transition of the emulsified high-density amorphous ice made by pressure-induced amorphization. J. Chem. Phys. 121, 3161-3164.

23) Mishima, O. and Stanley, H. E. (1998) Decompression-induced melting of ice IV and the liquid-liquid transition in water. Nature 392, 164-168.

24) Mishima, O. and Stanley, H. E. (1998) The relationship between liquid, supercooled and glassy water. Nature 396, 329-335.

25) Mishima, O. (2000) Liquid-liquid critical point in heavy water. Phys. Rev. Lett. 85, 334-336.

26) Fuentevilla, D. A. and Anisimov, M. A. (2006) Scaled equation of state for supercooled water near the liquid-liquid critical point. Phys. Rev. Lett. 97, 195702.

27) Bellissent-Funel, M.-C. and Bosio, L. (1995) A neutron scattering study of liquid $\mathrm{D}_{2} \mathrm{O}$ under pressure and at various temperatures. J. Chem. Phys. 102, $3727-3735$

28) Finney, J. L., Hallbrucker, A., Kohl, I., Soper, A. K. and Bowron, D. T. (2002) Structures of high and low density amorphous ice by neutron diffraction. Phys. Rev. Lett. 88, 225503.

29) Harrington, S., Zhang, R., Poole, P. H., Sciortino, F. and Stanley, H. E. (1997) Liquid-liquid phase transition: Evidence from simulations. Phys. Rev. Lett. 78, 2409-2412.

30) Martoňák, R., Donadio, D. and Parrinello, M. (2004) Polyamorphism of ice at low temperatures from constant-pressure simulations. Phys. Rev. Lett. 92, 225702.

31) Poole, P. H., Sciortino, F., Grande, T., Stanley, H. E. and Angell, C. A. (1994) Effect of hydrogen bonds on the thermodynamic behavior of liquid water. Phys. Rev. Lett. 73, 1632-1635.

32) Buldyrev, S. V., Malescio, G., Angell, C. A., Giovambattista, N., Prestipino, S., Saija, F. et al. (2009) Unusual phase behavior of one-component systems with two-scale isotropic interactions. J. Phys.: Condens. Matter 21, 504106.

33) Aasland, S. and McMillan, P. F. (1994) Densitydriven liquid-liquid phase separation in the system $\mathrm{Al}_{2} \mathrm{O}_{3}-\mathrm{Y}_{2} \mathrm{O}_{3}$. Nature 369, 633-636.

34) Katayama, Y., Mizutani, T., Utsumi, W., Shimomura, O., Yamakata, M. and Funakoshi, K. (2000) A first-order liquid-liquid phase transition in phosphorus. Nature 403, 170-173.

35) Debenedetti, P. G. (2003) Supercooled and glassy water. J. Phys.: Condens. Matter 15, R1669-R1726.

36) Ball, P. (2001) Life's matrix: a biography of water. University of California Press, Berkeley and Los Angeles.

37) Liu, L., Chen, S. H., Faraone, A., Yen, C. W. and Mou, C. Y. (2005) Pressure dependence of fragileto-strong transition and a possible second critical point in supercooled confined water. Phys. Rev. Lett. 95, 117802.

38) Kim, C. U., Kapfer, R. and Gruner, S. M. (2005) High-pressure cooling of protein crystals without cryoprotectants. Acta Cryst. D61, 881-890.

39) Angell, C. A. and Sare, E. J. (1968) Liquid-liquid immiscibility in common aqueous salt solutions at low temperatures. J. Chem. Phys. 49, 4713-4714.

40) Suzuki, Y. and Mishima, O. (2000) Two distinct Raman profiles of glassy dilute $\mathrm{LiCl}$ solution. Phys. Rev. Lett. 85, 1322-1325.

41) Mishima, O. (2005) Application of polyamorphism in water to spontaneous crystallization of emulsified LiCl- $\mathrm{H}_{2} \mathrm{O}$ solution. J. Chem. Phys. 123, 154506.

42) Mishima, O. (2007) Phase separation in dilute $\mathrm{LiCl}$ $\mathrm{H}_{2} \mathrm{O}$ solution related to the polyamorphism of liquid water. J. Chem. Phys. 126, 244507.

43) Koop, T., Luo, B., Tsias, A. and Peter, T. (2000) Water activity as the determinant for homogeneous ice nucleation in aqueous solutions. Nature 406, 611-614.

(Received Dec. 14, 2009; accepted Jan. 16, 2010) 


\section{Profile}

Osamu Mishima was born in Hiroshima in 1951. He learned the high-pressure science and technology at Faculty of Engineering Science, Osaka University, and studied the high-pressure phases of ice. He received his B. Eng., M. Eng., and Dr. Eng. from Osaka University in 1974, 1976, and 1979, respectively. After he served as an Assistant at the Osaka University Faculty of Engineering Science's High-Pressure Laboratory, he became a Research Associate at National Research Council of Canada in Ottawa. He discovered both the pressure-induced amorphization of crystalline ice and the polyamorphism of amorphous ice with collaboration of Dr. E. Whalley and Dr. L. D. Calvert. These discoveries have started to change the view of water and to provide a much broader perspective on liquid and glass. He joined National Institute for Researches in Inorganic Materials (NIRIM) in Tsukuba in 1985. He continued

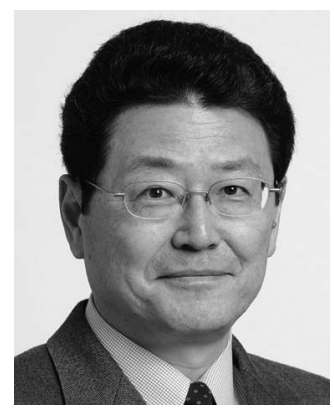
the studies on the polyamorphism of water while he made at a high pressure the first primitive ultraviolet-lightemitting pn-junction diode of cubic-boron-nitride (cBN) semiconductor. At NIRIM, and at National Institute for Materials Science (NIMS) since 2001, he focused his studies on the water researches as an Independent Senior Researcher. He received the Nishina Memorial Prize in 2006, and was named NIMS Fellow in 2007. He received the Japan Society of High Pressure Science and Technology Award in 2002 and the Commendation for Science and Technology by the Minister of Education, Culture, Sports, Science and Technology (Prizes for Science and Technology, Research Category) in 2008. 